\title{
Tratamento de seroma causado por intercorrência em bichectomia
}

\author{
Treatment of seroma caused by complications in bichectomy
}

Tratamiento del seroma causado por complicaciones en la bichectomía

Mário Silveira de Souza ${ }^{1 *}$, Célia Marisa Rizatti Barbosa ${ }^{1,2}$, Roberto Pontes Dias de Oliveira ${ }^{3}$, Daniela Martins de Souza 4 .

\section{RESUMO}

Objetivo: Apresentar o relato de um caso de proposta terapêutica para seroma, diagnosticado por ecografia, que se desenvolveu após dez dias da cirurgia de bichectomia em paciente de 35 anos do gênero feminino. Detalhamento do caso: A indicação estética de bichectomia foi realizada para delineamento facial, ressaltando contornos do rosto e promovendo jovialidade. Diversas intercorrências podem ser observadas neste tipo de procedimento, no entanto, o diagnóstico de seroma parece apresentar raridade. Após dez dias, observou-se presença de grande edema, de consistência rígida, na região da cirurgia do lado esquerdo. A paciente foi tratada com antibiótico e drenagens do exsudato até o completo colabamento das paredes internas da lesão. Considerações finais: A ocorrência de seroma após bichectomia pode estar associado a focos prévios de infecção bucal e ao uso de eletrocautério e que a proposta terapêutica por meio de antibioticoterapia por 20 dias associada a drenagens periódicas conforme a formação do edema por exsudato resultou em cura da lesão e manutenção da saúde.

Palavras-chave: Rejuvenescimento, Tecido adiposo, Assimetria facial.

\section{ABSTRACT}

Objective: Present a case report of a therapeutic proposal for seroma, diagnosed by ultrasound, which developed ten days after a bichectomy surgery in a 35-year-old female patient. Details of the case: The aesthetic indication for bichectomy was performed for facial design. Several complications can be observed in this type of procedure, however, the diagnosis of seroma seems to present rarity. After ten days, the presence of a large edema was observed in the region of the surgery on the left side. The patient was treated with antibiotics and drainages of the exudate until the complete collapse of the internal walls of the lesion. Final considerations: That the occurrence of seroma after bichectomy may be associated with previous focuses of oral infection and the use of electrocautery and that the therapeutic proposal by means of antibiotic therapy associated with periodic drainages resulted in healing of the lesion.

Keywords: Rejuvenation, Adipose tissue, Facial asymmetry.

\section{RESUMEN}

Objetivo: Presentar un informe de un caso con una propuesta terapéutica para el seroma, diagnosticado a través de un examen de ecografía, que se desarrolló diez días después de realizada una cirugía de bichectomia en un paciente de 35 años de género femenino. Detalles del caso: Se indicó con base a la estética, la realización de bichectomía para el delineamiento facial. Se pueden observar varias complicaciones en este tipo de procedimiento, sin embargo el diagnóstico de seroma suele ser raro. Después de 10 días, se observó que surgió un gran edema en la región donde fue realizada la cirugía del lado izquierdo. La paciente

\footnotetext{
${ }^{1}$ Centro Universitário (UNINGÁ), Maringá - Paraná. *E-mail: mario@harmoniza.com.br

2 Universidade Estadual Paulista Júlio de Mesquita Filho. São Paulo - SP.

${ }^{3}$ Radiologia e Diagnóstico por Imagem (UNIMINAS), Porto Alegre - RS.

${ }^{4}$ Faculdades Unidas do Norte de Minas (FUNORTE), Montes Claros - MG.
} 
fue tratada con antibióticos y drenaje del exudado hasta la mejora completa de las paredes internas de la lesión. Consideraciones finales: La aparición de seroma después de la realización de una bichectomía puede estar asociada a la presencia de focos previos de infección oral y al uso de electrocauterización y que la propuesta terapéutica de prescripción de antibióticos y asociada a drenajes periódicos resultó en la cura de la lesion.

Palabras clave: Rejuvenescimento, Tecido adiposo de bichat, Assimetria facial.

\section{INTRODUÇÃO}

No contexto histórico a "Bola de Bichat" foi descrita primeiramente por Laurentius Heister como estrutura de natureza glandular denominada "Glândula Molar" devido à sua proximidade com os dentes molares (ROUVIÈRE H e DELMAS A, 2005). Sete décadas depois, Marie François Xavier Bichat, atribuiu o seu sobrenome à estrutura, Bola de Gordura de Bichat, reclassificando-a como tecido adiposo e não glandular (GRÖBE A, et al., 2011). Constatou-se sua estabilidade nos casos de perda de peso, independentemente da quantidade de gordura corporal do indivíduo (NICOLICH F e MONTENEGRO C, 1997).

Egyedi P (1977), descreveu a técnica de fechamento de comunicações buco sinusal, usando a gordura de Bichat de forma pediculada, e o procedimento se tornou útil em cirurgias odontológicas. A partir da década de 80 vários profissionais recorreram ao uso da gordura de Bichat para tratar comunicações oro-antrais de diversas etiologias: agudas, crônicas ou recorrentes (FARIA CADC, et al., 2018).

lindicação estética aumentou nos últimos anos, com objetivo de delinear o rosto, evidenciando os ângulos e contornos faciais, proporcionando aspecto de emagrecimento e ressaltando as características esqueléticas da face (FARIA CADC, et al., 2018).

A função bucal da Bola de Bichat é de proteger as estruturas anatômicas sensíveis próximas, como vasos e nervos, além de participar da função de sucção, evitando o colabamento dos músculos bucinadores e da função mastigatória, evitando atrito e sobrecarga nos músculos e estruturas envolvidas (BRUCOLI M, et al., 2011). Sua importância funcional está mais relacionada a fase de amamentação onde a sucção é fundamental para o desenvolvimento da criança e onde importantes estruturas anatômicas ainda não se encontram protegidas pelo desenvolvimento músculo esquelético (EGYEDI P,1977; BRUCOLI M, et al., 2011).

Anatomicamente o tecido gorduroso de Bichat é composto de massa adiposa compartilhada em lóbulos, com corpo central e quatro extensões: bucal, pterigóide, maxilar e temporal (EGYEDI P,1977). A porção diretamente associada a estética e função facial é a porção bucal, que está localizada abaixo e à medial do ducto parotídeo, posterior à saída do ducto de Stenon, entre os músculos bucinador e a porção anterior do músculo masseter. Ao seu limite superior, observa-se região zigomática, e no limite cirúrgico anatômico, na região anterior, está a veia e artéria facial, que devem ser preservadas na incisão, bem como o ducto de Stenon em seu limite superior (EGYEDI P,1977; ALMEIDA A e ALVARY P, 2018).

A Gordura de Bichat apresenta volume médio de 9,5 a $10 \mathrm{ml}$, o que equivale aproximadamente a $9 \mathrm{~g}$, podendo haver divergência de tamanho entre os lados direito e esquerdo do paciente. No entanto, a porção bucal, apresenta volume e peso médio menor que a metade do volume e peso total da estrutura, variando entre 3 e $4 \mathrm{ml}$ de gordura (JACOMETTI V, et al., 2017).

O metabolismo do corpo adiposo de Bichat, assim como da gordura orbitária, é diferente do comportamento metabólico de outros compartimentos adiposos. Dessa forma, pacientes bariátricos ou que perderam muito peso e massa corporal, podem ter indicação estética para diminuir o aspecto arredondado ou ptosado da face (FARIA CADC, et al., 2018).

O procedimento cirúrgico para remoção parcial do tecido gorduroso da bochecha, bichectomia, tem indicação estética, funcional ou estético-funcional. A atividade mastigatória lesiva, como apertamento cêntrico e/ou excêntrico, resulta em mordiscamento da mucosa jugal, ocasionando dor, desconforto e proeminência hiperplásica da linha alba na região interna da bochecha (ALMEIDA A e ALVARY P, 2018). Nessas condições clínicas, podem ocorrer lesões importantes na mucosa bucal, além de agressões a todo o sistema 
estomatognático, o que indica a bichectomia como alternativa de tratamento complementar (RIZZATTIBARBOSA CM, et al., 2017, RIZZATTI-BARBOSA CM, 2019).

A cirúrgia é relativamente simples e as complicações, quando ocorrem, podem ser no transoperatório ou no pós-operatório. As intercorrências podem ser de graves consequências e difícil resolução, mesmo para os profissionais mais experientes (MOREIRA Junior R, et al., 2018). As complicações transitórias comumente evidenciadas são: hematomas, abcessos, parestesia transitória e trismo. Raramente ocorre complicações severas como paralisia facial por ruptura dos ramos bucal e zigomático do nervo facial, estenose do ducto parotídeo por ruptura ou assimetria facial decorrente da remoção bilateral em quantidades distintas. A bichectomia quando realizada por profissional experiente diminui as chances de intercorrência (ALMEIDA A e ALVARY P, 2018; MOREIRA Junior R, et al., 2018).

Após procedimentos semelhantes a bichectomia, como mastectomia, o local poderá infeccionar, o que irá complicar a cicatrização, podendo desenvolver seroma, lesão inflamatória exsudativa de baixa prevalência (McCAUL JA, et al., 2000). O objetivo desse trabalho foi descrever por meio de caso clínico, a ocorrência e os fatores etiológicos, bem como a proposta terapêutica da lesão tipo seroma, manifestada após cirurgia de bichectomia.

\section{DETALHAMENTO DO CASO}

A paciente foi previamente informada sobre o procedimento a ser realizado e possíveis complicações que poderiam ocorrer. Caso houvesse qualquer intercorrência a mesma seria prontamente tratada até seu restabelecimento. A paciente concordou em realizar o procedimento, assinou o Termo de Consentimento Livre e Esclarecido e o Termo de autorização de uso de imagem em eventos e atividades científicas.

Paciente SRO, gênero feminino, 35 anos, brasileira, sem comorbidades. Apresentava necessidade de tratamento odontológico para lesões cariosas, raspagem supra gengival e extrações de restos radiculares. Além de interesse em realização do procedimento de Bichectomia com finalidade essencialmente estética. Ao exame extraoral, observa-se imagem inicial frontal da paciente (Figura 1). O ato cirúrgico da bichectomia transcorreu sem intercorrências, apresentou duração aproximada de 60 min entre a anestesia e a sutura final. A incisão foi realizada com bisturi eletrônico BP-100 Plus, eletro cautério, marca EMAI - Transmai (Brasil). Durante o procedimento cirúrgico, observou-se posicionamento superior da porção bucal da bola de Bichat, resultando em maior manipulação para dissecção dos tecidos. Removeu-se $4 \mathrm{ml}$ de material adiposo de Bichat de ambos os lados (Figura 2).

Figura 1 - Exame extraoral inicial frontal.

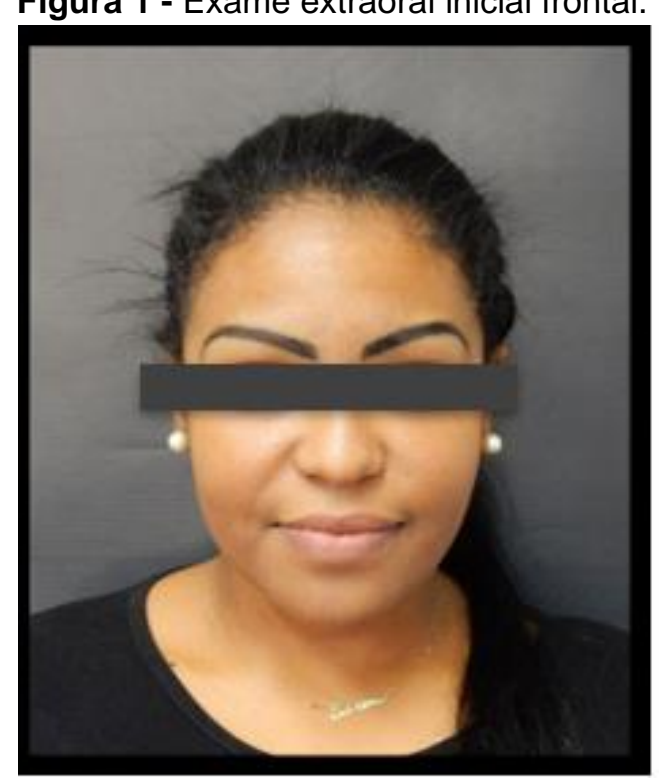

Fonte: Souza MS, et al., 2020.

REAS/EJCH | Vol.12(11) | e4272 | DOI: https://doi.org/10.25248/reas.e4272.2020 Página 3 de 8 
Figura 2 - Porção Bucal da Bola de Bichat.

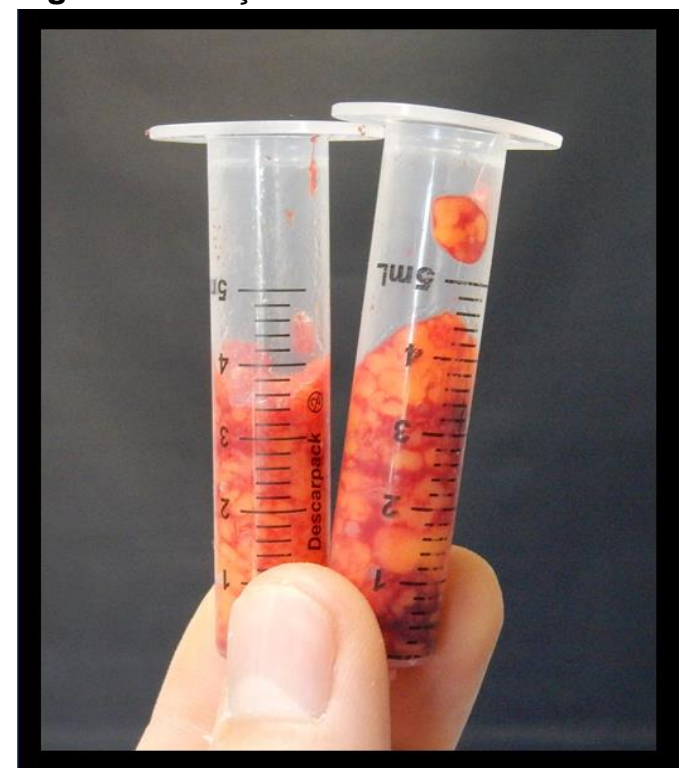

Fonte: Souza MS, et al., 2020.

Após a cirurgia houve ausência de dor, hematoma ou parestesia. Tendo o pós-operatório imediato transcorrido normalmente para esse tipo de procedimento. Foi prescrito antibiótico, anti-inflamatório e analgésico como procedimento de rotina, e não foram utilizadas faixas compressoras. Após dez dias, observou-se presença de grande edema, de consistência rígida, na região da cirurgia do lado esquerdo (Figura 3). Foi substituída a antibioticoterapia inicial pós-cirúrgica, de Amoxicilina 500 mg + Ácido Clavulânico 125mg de 8 em 8 horas por 7 dias, por Clindamicina 300 mg + Metronidazol 400 mg, por mais 10 dias. Foi prescrito também Dexametazona, por 5 dias.

Figura 3 - Edema após 10 dias do procedimento.

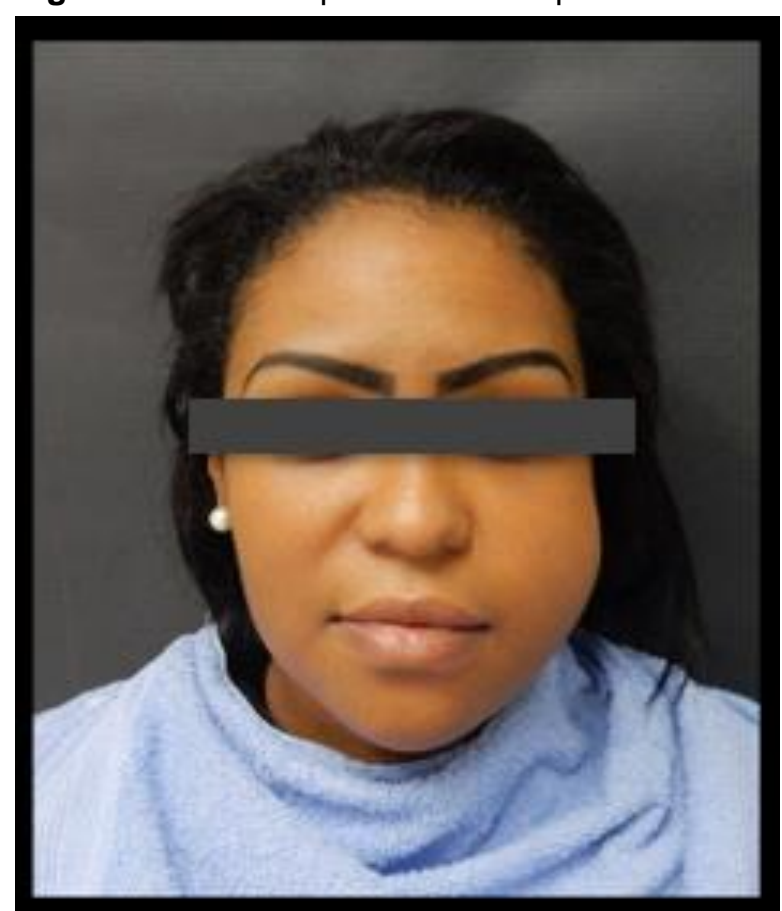

Fonte: Souza MS, et al., 2020.

Após 14 dias da cirurgia ocorreu a drenagem de material líquido, viscoso e purulento, resultando redução de volume do abscesso. Transcorrido mais 4 dias após a primeira drenagem novo exame clinico constatou 
redução do edema e nova drenagem ocorreu após ordenha, porém de líquido hialino e sem a presença de supuração.

Sob suspeita de ruptura do ducto de Stenon, recorreu-se ao exame complementar de imagem. O diagnóstico da lesão foi obtido por aparelho de ultrassonografia, marca GE modelo P6 (Alemanhã), na região afetada. Na imagem, observou-se presença de seroma no lado esquerdo, medindo $14 \mathrm{~mm} \times 24 \mathrm{~mm}$ e com volume de $3,8 \mathrm{ml}$. Neste mesmo exame foi verificada a integridade do Canal de Stenon e da Glândula Parótica. Na Figura 4, observa-se o lado direito, com a cicatrização normal, e o lado esquerdo, com a presença do seroma.

Figura 4 - Lado direito da paciente Normal (D/N) e o lado esquerdo da paciente com Seroma (E/S).

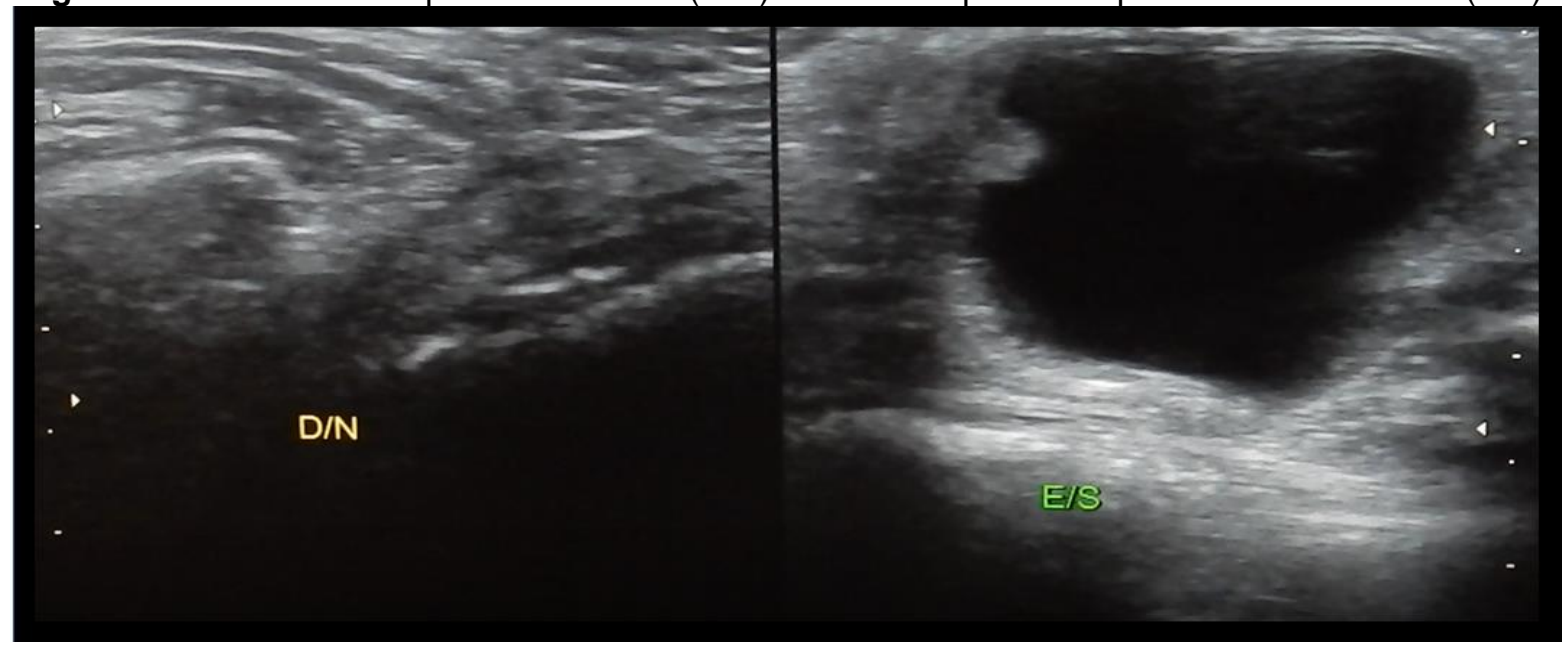

Fonte: Souza MS, et al., 2020.

Seguido de mais dois procedimentos de drenagem em ambulatório e sob anestesia local, com intervalo aproximado de 5 dias entre eles, houve completa remissão dos sinais e sintomas do seroma (Figura 5).

Figura 5 - Drenagem do seroma.

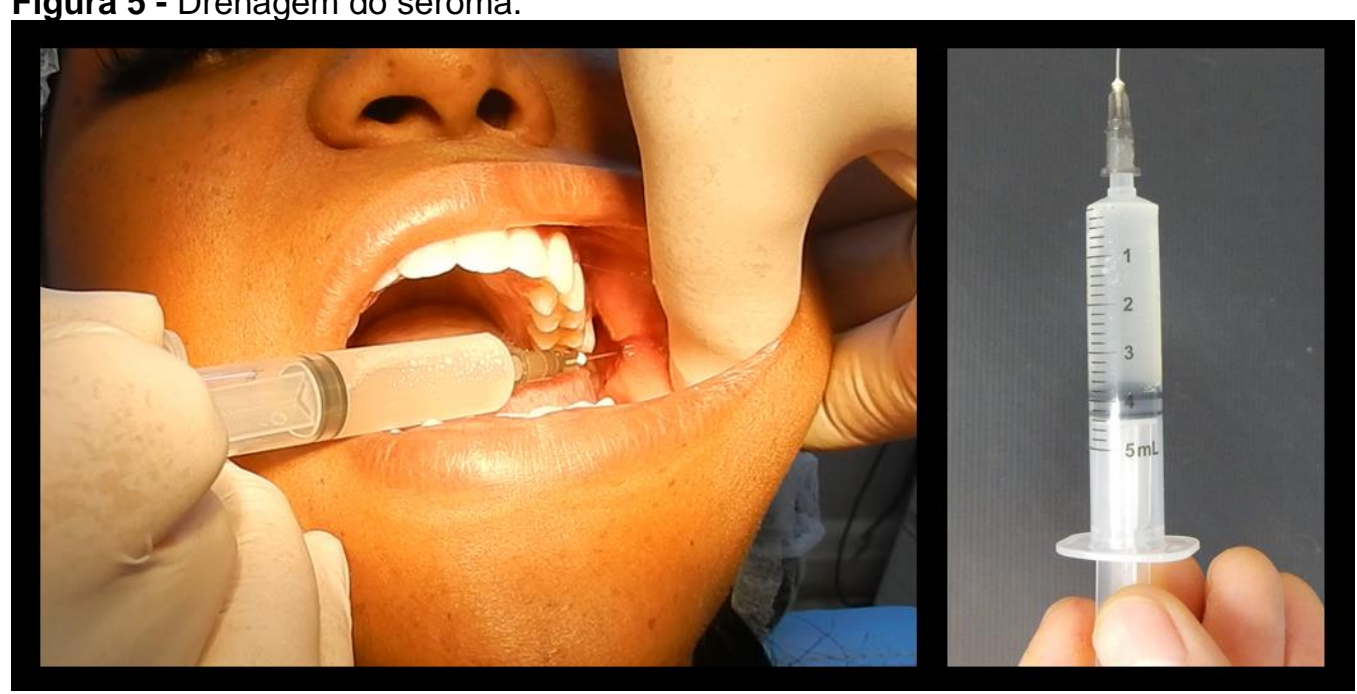

Fonte: Souza MS, et al., 2020.

O Líquido coletado foi enviado para análise citológica e o resultado apresentou esfregaços constituídos por linfócitos e macrófagos, sem presença de células malignas. A paciente permaneceu em acompanhamento clinico. Na Figura 6, observa-se no início do caso o aspecto clinico frontal, meio perfil lado afetado pelo seroma e lateral do lado afetado pelo seroma. Na mesma figura, na parte de abaixo, observa-se nas mesmas possições após transcorridos 94 dias do tratamento. 
Figura 6 - Imagens $1 A, 1 B$ e $1 C$ fotos iniciais. Imagens $2 A, 2 B$ e $2 C$ fotos após 94 dias, finalização do caso.

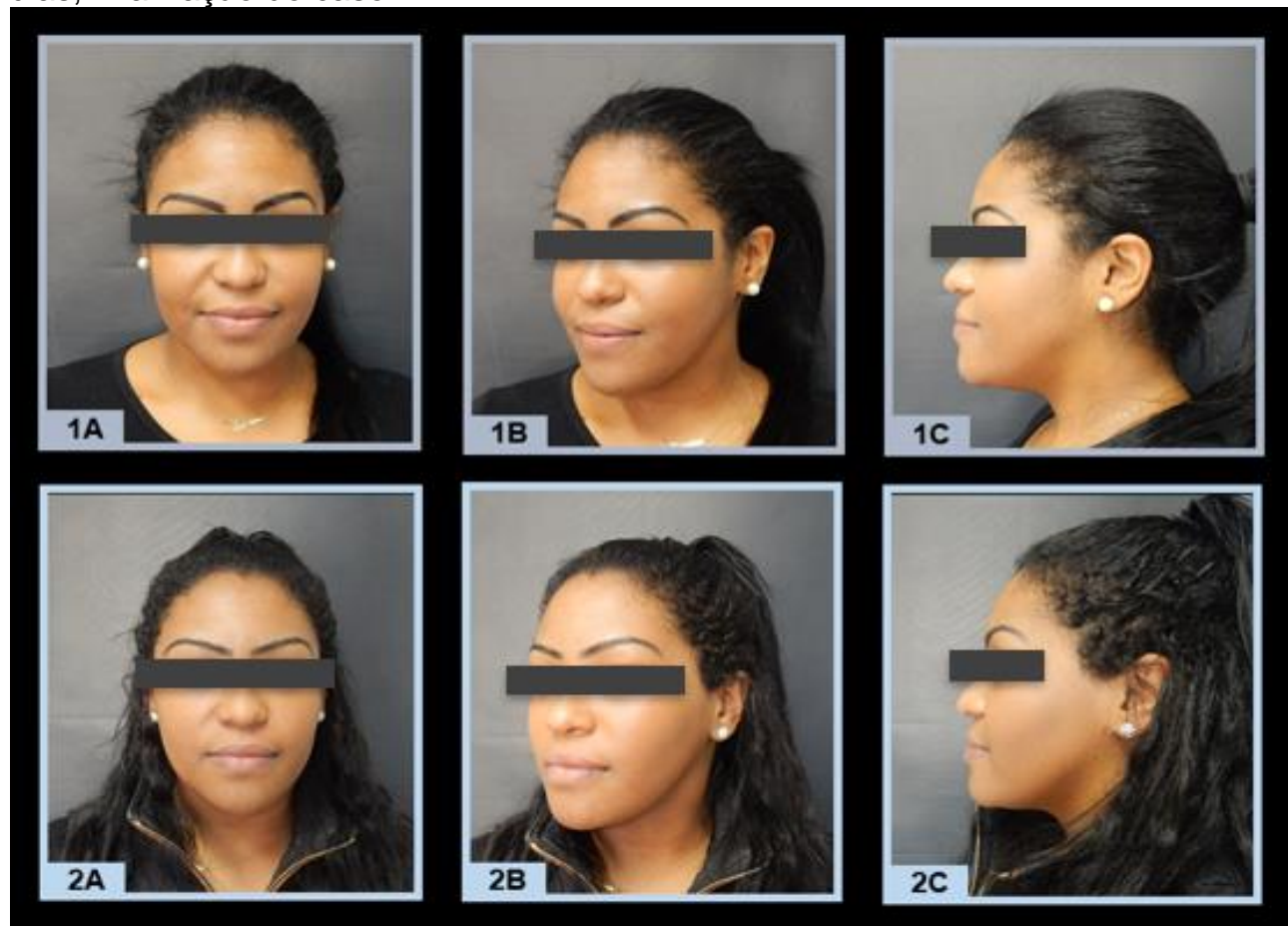

Fonte: Souza MS, et al., 2020.

\section{DISCUSSÃO}

O presente caso clínico relatou a possiblidade do desenvolvimento de lesão do tipo seroma após cirurgia de bichectomia, alertando para um tipo de intercorrência resultante, em parte, devido a negligencia da realização previa do tratamento odontológico ao procedimento de remoção do corpo adiposo de Bichat. $\mathrm{O}$ relato propõe precauções que evitariam sua manifestação e apresenta proposta terapêutica bem sucedida para cura e manutenção da saúde.

Vários fatores têm sido correlacionados com a formação de seroma, porém faltam evidencias para comprovar quais destes fatores tem relação com a sua etiologia, o que dificulta a identificação dos pacientes que poderão desenvolver a lesão (McCAUL JA, et al., 2000).

Estudo prospectivo e randomizado de mastectomia, demonstrou elevada prevalência de ocorrência de lesão inflamatória tipo seroma quando a incisão foi realizada com eletrocautério em relação às incisões convencionais com bisturi (PORTER K, et al., 1998). No presente estudo a incisão foi realizada com eletrocautério, o que pode ter interferido para o desenvolvimento dessa lesão. Podemos supor que o uso do eletrocautério nas cirurgias de bichectomia, contribui como fator predisponente para o desenvolvimento de seroma como intercorrência cirúrgica.

Os seromas são geralmente indolores e sua ocorrência tem sido relacionada a infecções pós operatórias e complicações na cicatrização. Após procedimentos de mastectonia e lipoplastia abdominal, que se assemelham a bichectomia, o local poderá infeccionar podendo desenvolver essas lesões inflamatórias exsudativas (McCAUL JA, et al., 2000, KUROI K, et al., 2006). No presente trabalho, a paciente apresentava focos de infecções dentais e periodontais, sendo postergado a raspagem supra gengival, restaurações das lesões cariosas e a exodontia dos restos radiculares dos dentes 37 e 38.

Observou-se que os maiores focos de contaminação oral estavam presentes no mesmo lado que se desenvolveu o seroma, indicando associação da lesão com a infecção prévia a cirurgia. Desta forma acreditamos que o reestabelecimento da saúde oral deve ser prioridade a fim de evitar intercorrências após a bichectomia. Sugerimos inclusão da possibilidade desta intercorrência e de suas implicações no termo de consentimento livre e esclarecido ao procedimento de bichectomia. 
Os processos infecciosos dento-alveolares podem se expandir pelos tecidos que oferecerem menor resistência. A propagação dos abscessos dentários em relação à ocorrência de fístulas intra-oral ou extraoral é explicado pelo arranjo dos músculos, considerando as relações anatômicas das linhas de inserção destes músculos com o processo alveolar (DUBRULL EL, 1991; SOUZA DM, et al., 2004). A exteriorização vestibular de abcessos de origem nos molares inferiores não é comum, devido a barreira imposta pela inserção e fáscia do músculo bucinador. (COHENCA N, et al., 2003).

Especificamente, no presente estudo a pré-existência de restos radiculares dos dentes 37 e 38 parece ter sido um fator relevante na contaminação do processo cicatricial. Além disso, durante a bichectomia a incisão de acesso foi realizada no músculo bucinador, criando-se dessa forma um canal de comunicação para infecção da ferida cirúrgica à distância.

A lesão do tipo seroma apresenta, em relação à sua composição bioquímica e parâmetros hematológicos, grande presença de granulócitos e monócitos indicando que o líquido do seroma represente secreções das fases exsudativas da resposta inflamatória.

Essa lesão não é composta por coleção plasmática e nem linfática, embora haja contaminação por elementos sanguíneos na sua composição (McCAUL JA, et al., 2000). Evidenciamos no relato do caso a presença de exsudato purulento nas primeiras drenagens e após antibioticoterapia de exsudato hialino composto por linfócitos e macrófagos. Assim sendo, preconizamos antibioticoterapia em qualquer tipo de seroma, pois a comunicação necessária para as drenagens pode contaminar a lesão, dificultando a cicatrização final.

O tratamento do seroma é realizado por procedimento clínico ambulatorial, podendo ser sob anestesia local ou mesmo sem anestesia, por meio de drenagens frequentes até o colabamento das paredes internas da lesão. Ressaltamos a importância das drenagens uma vez que, Nahas FX, et al. (2007); alertaram que se o seroma não for drenado e o fluído permanecer por longo período, pode encapsular entorno da lesão gerando deformidade secundária. Neste caso, cirurgia estética de correção será necessária.

A conduta terapêutica adotada foi: a partir da confirmação do diagnóstico de seroma, por meio de ecografia, administração de antibioticoterapia por 10 dias associada a drenagens semanais, conforme redução do edema, até o complete colabamento das paredes internas do seroma em definitivo. A resolução da intercorrência de seroma, neste caso clínico, evoluiu positivamente sem nenhum tipo de sequela ao paciente.

Após os resultados desse caso clínico, sugere-se necessidade de realização de futuros estudos que avaliem a interferência de faixas compressivas e a auto massagem de drenagem, por parte do paciente, como benefícios no processo cicatricial. Além disso o uso de sutura parcial com a finalidade de proporcionar drenagem espontânea para prevenir a ocorrência de seroma. A técnica de bichectomia com finalidade estética ganhou notoriedade em vários países nos últimos anos, tornando-se uma tendência principalmente entre as mulheres (MOREIRA JUNIOR R, et al., 2018). No contexto da Harmonização Orofacial e devido a popularidade desse procediemto necessita-se de mais pesquisas e publicações científicas.

A ocorrência de seroma após procedimento de bichectomia pode estar associado a focos prévios de infecção bucal e também a utilização de eletrocautério na realização da cirúrgia. E a proposta terapêutica por meio de antibioticoterapia por 20 dias, associada a drenagens periódicas conforme a formação do edema por exsudato resultou em cura da lesão e manutenção da saúde.

\section{REFERÊNCIAS}

1. ALMEIDA A, ALVARY P. A Bichectomia como procedimento Cirúrgico Estético-Funcional: Um estudo crítico: Case report. J Business Techn. 2018;7(1):3-14.

2. BRUCOLI M, et al. Surgical technique of the transoral pproach to remove a lipoma of the buccal fat pad. J Craniofac Surg, 2011; 22(6): 2415-18.

3. COHENCA N, et al. Extraoral sinus tract misdiagnosed as endodontic lesion. J. Endod, 2003; 29 (12): 841-3.

4. DUBRULL EL. Anatomia Oral de Sicher e Dubrul. 8.ed. São Paulo: Artes médicas. 1991. 377 p.

REAS/EJCH | Vol.12(11) | e4272 | DOI: https://doi.org/10.25248/reas.e4272.2020 Página 7 de 8 
5. EGYEDi P. Utilization of buccal fat pad for closure of oroantral and/or oronasal communications. J. Maxillofacial Surg, 1997; 5(4): 241-244.

6. FARIA CADC, et al. Bichectomia e sua contribuição para harmonia facial. 2018 Bichectomia e sua contribuição para harmonia facial Rev. Bras. Cir. Plást. 2018; 33(4):446-452

7. GRÖBE A, et al. The use of buccal fat pad (BFP) as a pedicled graft in cleft palate surgery. Int J Oral Maxillofac Surg, 2011; 40: 685-689.

8. JACOMETTI V, et al. Procedimento de bichectomia: uma discussão sobre os aspectos éticos e legais em odontologia. Rev. Brasileira de cirurgia plástica; 2017.34(4):616-23.

9. KUROI K, et al.: Evidence-Based Risk Factors for Seroma Formation in Breast Surgery. 2006

10. McCAUL JA, et al. Aetiology of seroma formation in patients undergoing surgery for breast câncer. UK, 2000.

11. MOREIRA JUNIOR R, et al. Bichectomia, a simple and surgery: case report. Rev Odontol Bras Central, 2018; 27(81): 98-100.

12. NAHAS FX, et al.: Does Quilting Suture Prevent Seroma in Abdominoplasty? São Paulo, Brasil, 2007.

13. NICOLICH F, MONTENEGRO C. Extracción de La bola de Bichat: Uma operação simples com surpreendentes resultados. Folia Dermatológica Peruana, 1997; 8(1):1- 5.

14. PORTER $\mathrm{K}$ et al. Electrocautery as a Factor in Seroma Formation Following Mastectomy. The American Journal of Surgery, 1998; $176(7): 8-11$.

15. ROUVIEERE H, DELMAS A. Anatomía humana, descriptiva, topográfica y funcional. 11va ed. España: Editorial Elseiver Mansson; 2005.

16. RIZZATTI-BARBOSA CM, et al. Therapeutic effectiveness of a combined counseling plus stabilization appliance treatment for myofascial pain of the jaw muscles: A pilot study. Cranio, 2017; 35 (3):180-6.

17. RIZZATTI-BARBOSA CM. Etiologia das Disfunções Temporomandibulares In Marchini L, Santos JFF. Oclusão Dentária, 2 ed., Ed. Elsevier, Rio de Janeiro, 2019.

18. SOUZA DM, et al. Expansão e disseminação das lesões pelos tecidos bucais. Rev.Bbiociên., Taubaté. 2004;10 (4): 215-221. 